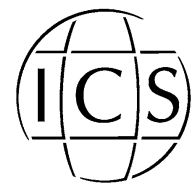

www.ics-elsevier.com

\title{
Development of a national monitoring protocol (developmental guide) for children with visual impairment 0-36 months
}

\author{
Alison Salt*, Naomi Dale, Jackie Osborne, Valerie Tadic \\ Developmental Vision Team, Great Ormond Street Hospital for Children NHS Trust/Institute of Child Health, \\ London and Department for Education and Skills, UK
}

\begin{abstract}
Objective: The aim of this project is to provide a national developmental monitoring protocol for infants and young children (0-36 months) with severe visual impairment (VI) that is underpinned by a scientific developmental framework. The profile will be used to inform and assist parents to help their baby progress and to recognise and, where possible, overcome areas of vulnerability in conjunction with their specialist teacher for the visually impaired and other professionals. Design: The work of our group and others has shown that infants with severe visual impairment are a particularly vulnerable group within the population of children with disability. They require a developmental profile that is specific to their developmental patterns and needs. The protocol is designed to provide practical guidance for parents and their keyworker on activities to promote the development of all skills. The development of the protocol draws on our specialist team's clinical experience, recent research and other developmental scales for young children with visual impairment (e.g. Oregon) and is informed by focus groups and piloting with parents and qualified teachers of children with visual impairment. Results: We describe the justification of the developmental framework, the outcome of focus groups with parents and specialist teachers and plans for implementation including the strategy for a local and regional health and education service framework for early intervention. Conclusions: This paper describes the first stage of development of this national monitoring protocol and guidance for parents of young children with visual impairment which will have important implications for services both nationally and internationally. (c) 2005 Published by Elsevier B.V.
\end{abstract}

Keywords: Monitoring protocol; Children; Visual impairment; Development

* Corresponding author. Tel.: +44 2078377618.

E-mail address: a.salt@ich.ucl.ac.uk (A. Salt). 


\section{Introduction}

The aim of this project is to provide a developmental monitoring protocol for infants and young children (0-36 months) with severe visual impairment (VI) that is underpinned by a scientific developmental framework. The profile will be used to inform and assist parents to help their baby progress and to recognise and, where possible, overcome areas of vulnerability in conjunction with their specialist teacher for children with visual impairment and other professionals.

The project is supported by the Department for Education and Skills (England) as part of the Early Support Programme which aims to support multi-agency working and family focused care for children with disability.

The work of our group and others has shown that infants with severe visual impairment are a particularly vulnerable group and require a developmental profile that is specific to their developmental patterns and needs.

The development of the protocol draws on our clinical experience, recent research and other developmental scales for young children with visual impairment (e.g. Oregon).

\section{Background}

Our Developmental Vision team at Great Ormond Street Hospital for Children (GOSH) has been at the forefront of developing materials, guidance and service delivery for babies and infants with visual impairment over a period of 30 years.

The population of the Developmental Vision Clinic (DVC) is infants and young children with severe visual disorders, referred for specialist management of their development. Our clinic serves a national population which, in light of the rarity of congenital visual impairment in the UK (3-4 per 10,000), gives us unique access and insight into the needs of young children with visual impairment and their families.

The team is multidisciplinary with staffing from neurodevelopmental paediatrics, psychology, nursing, occupational therapy, speech and language therapy and optometry.

\subsection{Parental involvement and multi-agency collaboration}

Our clinic has pioneered partnership ways of supporting and assisting parents. We work with parents and involve them as the key partner in helping their baby, involving them fully in our assessments, goal planning and using family-held records [1].

Our team is committed to working in collaboration with health, education and other agency partners, and have received national and international recognition for work in developing multidisciplinary/multi-agency partnership schemes [2].

\subsection{Lessons learned about infants with severe visual impairment}

Through a series of research studies spanning 25 years, our team has demonstrated that impaired vision can impose far-reaching and complex constraints on early development including motor, cognitive, language and social development [3-5]. Constraints on any target skill are often cumulative, acting directly upon it and also on one or more of the skills that underpin its acquisition [4]. In recent years we have demonstrated that babies and young children with visual impairment are especially developmentally vulnerable and that help is needed from the earliest days [6]. A sizeable subgroup shows a severe and 
potentially irreversible 'developmental setback' or regressive disorder in their second to third year [6] with plateauing or loss of cognitive and language skills and increasing disorder of social communication skills/autism. We have also established early warning signs of developmental vulnerability $[7,8]$ and are currently developing guidance strategies for enhancing early development and overcoming constraints arising from visual impairment and reducing risk factors. Early monitoring and intervention is therefore essential for promoting optimal use of vision and development [9] and for reducing the negative impact of other risk factors.

\subsection{Development of clinical tools}

Previous members of the team have developed an integrated scheme of assessment and management for a range of areas of development including visual development and functional vision [9-15].

\section{Development of the new monitoring protocol}

The monitoring protocol will be developed to provide a structured sequential guide of expected developmental steps in young children with visual impairment aged 0 to 36 months. It will build and expand on our current guide [13], providing more finely graded steps of key developmental stages especially in the areas of object relationships and reasoning, communication and social development [5,7], language and meaning, play and learning, movement and mobility and self help skills. The protocol will be specifically designed to be used jointly by parents and their key worker, with the scope to record achievements and any concerns.

Expectation for developmental levels reached at each stage will be explicit but sensitively handled, ensuring that there will be clear trigger points to indicate when more intensive intervention is required.

The protocol will include: additional guidance for babies and children showing early developmental and behavioural difficulties; advice about common problems and special sections - 'shared discovery', visual promotion, simple functional vision assessment and promotion of pre-Braille skills.

Our recent research has demonstrated that children with 'potentially simple' congenital disorders of the peripheral visual system ('potentially simple' CDPVS) are the most appropriate subgroup for establishing developmental norms [5,7]. We have also shown that development is likely to proceed at different rates in infants who have 'form' vision compared with those without. The protocol will take account of these different expectations for development and provide differentiated practical strategies to help children with particular tasks. We have also demonstrated a simple standard test for measuring for 'form' vision [10-12], which can be satisfactorily administered and interpreted by staff of different disciplines after preliminary training.

The monitoring protocol will be an essential guide and framework for parents and their key worker. Because of the considerable diversity and variation in visual disorders in infants and young children with visual impairment, the profile should be used in the context of support from and comprehensive assessment by specialists, trained and experienced in visual impairment in infancy and childhood. 


\section{Messages from Parent focus groups}

Twelve parents of children with severe and profound visual impairment have been involved in three focus groups during the early stages of development of the protocol. They have provided invaluable ideas for the development of the protocol.

\subsection{Key messages}

- Early identification and early support for parents following diagnosis are essential elements of any programme prior to introducing such a protocol. The immense emotional needs of parents at the time of diagnosis must not be underestimated.

- Practical guidance for parents on how to assist their child in their learning must be an integral part of any protocol.

- The protocol needs to be simple to use and information easily accessible — not like 'a college course'. Video material would be welcome in addition to written material.

- Parents welcome expert support from professionals but the protocol needs to be able to provide 'stand alone' information and ideas in the event that an appropriate professional is not immediately available.

- Clear sign posting of goals and activities. Practical solutions to problems encountered, e.g. 'tips from other parents' would be welcome.

- There was ambivalence about explicit information about age related norms. For those children who were developing more slowly it was acknowledged that this could be distressing, but nevertheless parents concluded that knowledge of expected milestones for children with visual impairment compared to their sighted peers would be helpful for parents so that they had a full understanding of their child's development.

\section{Anticipated benefits}

The protocol will:

- help parents develop realistic informed expectations of early development in their child with visual impairment and give guidelines for expected ages for achieving milestones,

- provide practical ideas on the promotion of early developmental skills,

- provide a shared framework and support a joint collaboration between parents and local health and education professionals and specialist teams,

- help local professional advisors assess and monitor developmental progress,

- provide a developmental framework for shaping early monitoring and intervention,

- assist detection of earliest signs of developmental difficulties, thus enabling speedy referral to specialist health services when early developmental concerns occur,

- strengthen health - education collaboration in early childhood disability,

- lay the foundation for development of new infant and preschool.

Developmental scales for the VI population that meet modern psychometric and scientific standards [7] for which funding will be sought in 2004.

The protocol will benefit all children with visual impairment whether profound or less severe and children with additional and multiple disability and visual impairment 


\section{References}

[1] H. McConachie, et al., Giving assessment reports to parents, Archives of Disease in Childhood 63 (1988) $209-210$.

[2] N. Dale, Working with Families of Children with Special Needs: Partnership and Practice, Routledge, London, 1996.

[3] J. Reynell, Developmental patterns of visually handicapped children, Child: Care, Health and Development 4 (1978) 291-303.

[4] P.M. Sonksen, S.L. Levitt, M. Kitzinger, Identification of constraints acting on motor development in young visually disabled children and principles of remediation, Child: Care, Health and Development 10 (1984) $273-286$.

[5] P.M. Sonksen, N. Dale, Visual impairment in infancy: impact on neurodevelopmental and neurobiological processes, Developmental Medicine and Child Neurology 44 (2002) $782-791$.

[6] H. Cass, P.M. Sonksen, H. McConachie, Developmental setback in severe visual impairment, Archives of Disease in Childhood 70 (1994) 192-196.

[7] N. Dale, P.M. Sonksen, Developmental outcome, including setback, in young children with severe visual impairment, Developmental Medicine and Child Neurology 44 (2002) 613-622.

[8] M.C. Waugh, W.K. Chong, P.M. Sonksen, Neuroimaging in children with congenital disorders of the peripheral visual system, Developmental Medicine and Child Neurology 4 (1998) 812-819.

[9] J. Reynell, P. Zinkin, New procedures for developmental assessment of young children with severe visual handicaps, Child: Care, Health and Development 1 (1975) 61-69.

[10] P.M. Sonksen, The assessment of 'vision for development' in severely visually handicapped babies, Acta Ophthalmologica 157 (1983) 82-90 (Suppl.).

[11] P.M. Sonksen, A functional assessment of vision for intervention to facilitate general and visual development, Proceedings of the 8th Interactional Conference on Blind and Visually Impaired Children. International Council for Education of the Visually Impaired, 1993, pp. 33-40.

[12] P.M. Sonksen, A. Petrie, K.J. Drew, Promotion of visual development of severely visually impaired babies: evaluation of a developmentally based programme, Developmental Medicine and Child Neurology 33 (1991) 320-335.

[13] P.M. Sonksen, B. Stiff, Show me what my friends can see. A developmental guide for parents of babies with severely impaired sight and their professional advisors. London: The Institute of Child Health (1991, 1999).

[14] P.M. Sonksen, The assessment of vision in the preschool child, Archives of Disease in Childhood 68 (1993) $513-516$.

[15] P.M. Sonksen, J. Silver, The Sonksen-Silver Acuity System, Keeler Ltd., Windsor, 1988. 